\title{
Presenting the Board of Respiration: Annette Boehler
}

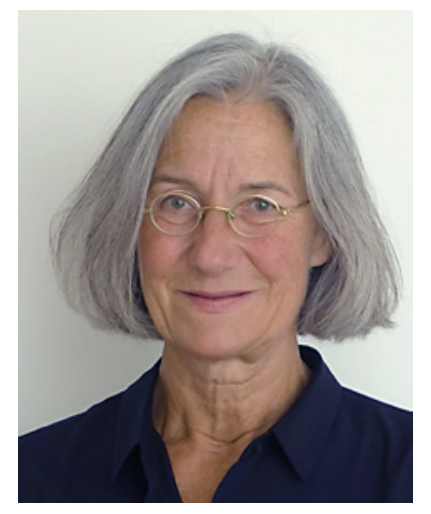

Prof. Annette Boehler is one of Respiration's Associate Editors. She is the former Medical Head of the Lung Transplant Program of the University Hospital Zurich (Switzerland), she is specialized in advanced lung diseases and lung transplantation.

\section{How Did You Become Interested in Advanced Lung Diseases and Lung Transplantation?}

After the initial years as resident in internal medicine, I started my specialization in pulmonary medicine right at the time when Zurich started its lung transplantation program. I was fascinated by the mixture of new opportunities in surgical techniques accompanied by pulmonary, infectious, and immunological challenges. The complexity of this field took me right in and in our small team - as in many centers around the world - the transdisciplinary spirit moved us forward. We learned everything from scratch much in analogy to kidney transplantation and in close collaboration with international pio- neer lung transplant programs (such as Papworth/UK and St. Louis/USA).

Early survival went well, but long-term results were less good, due to chronic allograft rejection in form of bronchiolitis obliterans.

Therefore, after board certification in internal and pulmonary medicine, I went to Papworth and later to Toronto to study this major complication of lung transplant recipients in depth. It was an amazing time in these visionary centers, and I got fascinating insights how these pioneer teams worked.

After my return to Switzerland, I became medical director of the lung transplant program, and, over the years, we developed a transplant program with excellent results based on a transdisciplinary team. In addition to peri- and posttransplant care, we formally established clinics for the specific lung diseases eventually leading to transplantation such as cystic fibrosis, interstitial lung diseases, and rare lung diseases. We further established diagnostics for these patient groups and offered all different therapies from conservative options to lung transplantation.

The close international scientific collaboration with leading programs around the world brought fascinating projects in basic and clinical research and opened new horizons also in this part of our field.

\section{Which Exciting Developments Are Currently Taking Place in This Field?}

On one hand, insights into the pathophysiology of chronic allograft rejection are increasing steadily. Contributing factors such as air pollution, allergens, viruses, 
etc. are being recognized in view of globalization and climate crisis.

There is much hope also in new pharmaceutic approaches with new drugs specific to certain lung diseases such as for pulmonary hypertension, interstitial lung diseases, and cystic fibrosis avoiding at all or at least postponing the final option of lung transplantation.

On the other hand, ex vivo lung perfusion is introduced by many centers leading to more and better donor organs. Preparing the lung-in-a-box to reestablish its function, ameliorate, and even regenerate it before transplanting it, opens a wide new horizon for organs initially considered of limited quality. This could substantially decrease the donor organ shortage in the near future.

And looking even further, approaches to solve the organ shortage with reassuring results include cartilaginous skeletons of lungs being seeded with the patient's own or allogeneic lung cells (semi-artificial lungs) leading to organ replacement by their semi-own lungs, a fascinating outlook for all transplant professionals.

\section{What Does the Future Hold for the Field, and What Do You Think Are the Main Challenges?}

An emerging future concept is organ repair clinics where lungs with advanced disease can be treated ex vivo while the patient is temporarily bridged on ECMO device before being retransplanted with his own cured lungs.

Another concept includes genetic modification of donor organs to avoid alloimmune responses.

Main challenges lay in resources: lung transplantation today requires complex lifelong follow-up and is in need of substantial resources in specialized healthcare. It is important to recognize that the best progress would be to prevent lung transplantation at all and to postpone advanced lung diseases by recognizing major contributing factors, by slowing down progression and by pharmaceutical stabilization of disease stages.

However, prevention might not be possible.

The lung is one of the most exposed organs to our environment, and globalization and climate crisis will impact in a multitude of facets: as seen today, with the Corona pandemic rising we might soon be witnessing a large number of cases of chronic impaired lung function in younger and older survivors with a totally new spectrum of patients.

\section{What Do You Feel Makes Respiration Special in the Publishing Landscape, and Are There Any Particular Papers You Would Like to See Submitted to the Journal?}

Our journal Respiration has a readership with a broad spectrum of interests. These interests and specialties are mirrored in the editorial board and associate editors who represent different fields of medicine from inside and outside of Europe.

In these days, there is concern about people caught in (scientific) bubbles and lack of communication in-between bubbles. In my opinion, our Journal is a good source to avoid this by covering the whole spectrum of scientific pulmonary medicine. Therefore, I am optimistic that our Journal also in the future stays at the frontline of medical advances. 